\title{
Emerging role of circular RNA in intervertebral disc degeneration: Knowns and unknowns (Review)
}

\author{
YONGJIN LI ${ }^{1 *}$, SUZHE ZHOU ${ }^{2 *}$, PENG PENG $^{3 *}$, XUKE WANG $^{1,4}$, \\ LILONG DU ${ }^{1}$, ZHENXIN HUO ${ }^{1}$ and BAOSHAN XU ${ }^{1}$ \\ ${ }^{1}$ Department of Minimally Invasive Spine Surgery, Tianjin Hospital, Tianjin 300211; \\ ${ }^{2}$ Department of General Practice, Xiamen Branch, Zhongshan Hospital, Fudan University, Xiamen, Fujian 361015; \\ ${ }^{3}$ Department of Orthopedics, Tianjin Medical University General Hospital, Tianjin 300052; \\ ${ }^{4}$ Department of Minimally Invasive Spine Surgery, Luoyang Orthopedic-Traumatological Hospital, \\ Luoyang, Henan 471002, P.R. China
}

Received February 1, 2020; Accepted June 4, 2020

DOI: $10.3892 / \mathrm{mmr} .2020 .11437$

\begin{abstract}
Lower back pain (LBP) is one of the predominant factors contributing to dyskinesia and remains a serious social and economic burden worldwide. Intervertebral disc degeneration (IDD) is the leading cause of LBP; the existing IDD treatments cannot completely prevent IDD. Circular RNAs (circRNAs) are non-coding RNAs resulting from back-splicing with unique structural characteristics and functions Accumulating evidence suggests that circRNAs are involved in the pathological process of IDD and modulate a range of IDD-related genes or proteins. However, the underlying circRNA-mediated regulatory mechanisms remain poorly understood. The aim of the present review is to describe the current understanding of circRNA characteristics, classification, biogenesis and function in relation to its specific roles in IDD. Additionally, the limitations on the current knowledge in the field and the future direction of IDD-related research are also discussed.
\end{abstract}

\section{Contents}

1. Introduction

2. Characteristics

3. Classifications

4. Biogenesis and functions

5. Specific roles of circRNA in IDD

Correspondence to: Professor Baoshan Xu, Department of Minimally Invasive Spine Surgery, Tianjin Hospital, 406 Jiefangnan Road, Hexi, Tianjin 300211, P.R. China

E-mail: baoshanxu99@tmu.edu.cn

${ }^{*}$ Contributed equally

Key words: intervertebral disc degeneration, circular RNA, nucleus pulposus cell, biofunction
6. Current limitations and future directions

7. Conclusions

\section{Introduction}

Lower back pain (LBP) is one of the predominant factors contributing to dyskinesia and the second most common cause of hospital visits, leading to a serious social and economic burden worldwide $(1,2)$. Symptomatic intervertebral disc degeneration (IDD) is the most frequent cause of $\operatorname{LBP}(1,3)$. Although several factors contribute to IDD, genetic factors are the leading cause (4). The nucleus pulposus (NP) is located in the center of the intervertebral disc (ID) (5); it is the largest avascular tissue in the body and lacks blood oxygenation, which limits its self-repairing ability (6). Existing IDD treatments are not satisfactory and cannot fully recover ID function (7). Non-coding RNA (ncRNA) generated by gene back-splicing can regulate gene post-transcriptional modification to modulate disease development (8). Frapin et al (9) described the pathological process of IDD in detail and inferred that metabolic dysregulation of the extracellular matrix (ECM) in the ID microenvironment was predominantly involved in the pathogenesis of IDD. The various types of IDD-related genes or protein expression disorders can contribute to the synthesis and catabolic imbalance of ECM, giving rise to the alteration of ID morphology, physics and mechanics, leading to ID function loss, thereby triggering IDD (9-11). Gene therapy uses viruses and other vectors to carry ncRNAs formed by genes or genes to target ID, which can reverse or block the pathological process of IDD and recover ID function at the genetic level (10). In view of the aforementioned reasons, gene-based diagnostic and treatment strategies are critical measures for IDD management.

IDD-related genes or proteins can be divided into protective factors and catabolic factors. The former includes hypoxia-inducible factor- $1 \alpha$ (HIF-1 $\alpha)$, vascular endothelial growth factor (VEGF), collagen type II (COL2), aggrecan (ACAN), SRY-related high mobility group box 9 (SOX9) and a series of antiapoptotic proteins, whereas the latter includes 
matrix metalloproteinases (MMPs), disintegrin and ADAM metallopeptidases with thrombospondin type 1 motifs (ADAMTSs), interleukin (IL)-1 $\beta$, tumor necrosis factor- $\alpha$ (TNF- $\alpha$ ) and a number of proapoptotic proteins (4,9-11).

Previous studies have suggested that ncRNAs, including microRNAs (miRNAs/miRs) and circular RNAs (circRNAs), serve a crucial role in the occurrence and progression of IDD (12-17). In particular, circRNAs mediate NP cell (NPC) apoptosis and regulate the expression of inflammatory cytokines, MMP, ADAMTS, various apoptosis-related proteins and key components of the ECM, such as COL2 and ACAN, which serve a role in the pathogenesis of IDD (12-17).

The present article provides an up-to-date review of circRNA characteristics, classification, biogenesis and function, with particular emphasis on the potential future directions of IDD-related research. Additionally, the limitations of current research are also discussed.

\section{Characteristics}

CircRNAs are a type of ncRNA with high thermal stability that were first discovered in plant-infecting virions in 1976 by Sanger et al (18). Although circRNAs have been identified in different species (19-22), these molecules were not initially extensively studied. However, circRNAs were later found to exert a previously unrecognized role in a wide spectrum of human diseases, owing to the rapid development of next-generation sequencing technology $(23,24)$. Compared with linear RNAs, covalently closed circRNAs have unique characteristics and biological functions without $5^{\prime}$ to $3^{\prime}$ polarity or a polyadenylated tail (25-27). They are predominantly located in the cytoplasm, abundantly expressed, conserved, highly stable and exonuclease-resistant (28-31). In addition, circRNAs are expressed in a tissue- and time-specific manner $(31,32)$. They can also be carried in exosomes and have potential applications as markers for disease diagnosis $(33,34)$. Direct back-splicing and exon skipping are the main pathways of circRNA synthesis $(23,27)$.

\section{Classifications}

Currently, seven types of circRNAs have been identified according to the type and quantity of the parental gene the circRNAs originate from. These include exonic circRNA (ecircRNA), intronic circRNA (ciRNA), tRNA intronic circRNA (tricRNA), exon-intron circRNA (eiciRNA), read-through circRNA (rt-circRNA), fusion circRNA (f-circRNA) and mitochondria-encoded circRNA (mecciRNA). The circularization of at least one intron or one exon from a single gene gives rise to ciRNAs and ecircRNAs, respectively, whereas the formation of eiciRNA is based on the cyclization of at least an exon and an intron $(24,26)$ (Fig. 1). TricRNA is a special type of circRNA synthesized through a pre-tRNA intron splicing mechanism (35). Rt-circRNA is the result of circularization of two exons from two different genes (30). F-circRNA is synthesized from the transcribed exons of several nuclear genes as a result of chromosomal translocation (36). Lastly, mecciRNA is produced from mitochondrial genes (37). EcircRNAs are the most common type of circRNA.

\section{Biogenesis and functions}

The biological functions of circRNAs depend on their type and cellular location. In the nucleus, synthetic circRNAs can modulate gene transcription $(30,38)$ and alternative splicing (39). For example, eiciRNAs circ-EIF3J and circ-PAIP2 are largely localized in the nucleus and can interact with U1 small nuclear ribonucleoprotein and RNA polymerase ( $\mathrm{Pol}$ ) II to promote the transcription of their host genes (38). The ciRNA ci-ankrd52 positively regulates the function of RNA Pol II to modulate the transcription of its parental gene (ANKRD52), predominantly converging to the cell nucleus (39). Increased synthesis of circ-Mbl may repress the transcription of its parental gene (40).

After synthesis, ecircRNAs are transferred from the nucleus to the cytoplasm. As shown in Fig. 1, the synthesis of mature miRNAs involves a range of processing steps. First, miRNA genes are transcribed into pri-miRNAs, and then processed into pre-miRNAs in the nucleus, pre-miRNAs are then transported into the cytoplasm via the nuclear export protein Exportin5 to produce mature miRNAs (41). Subsequently, mature miRNAs directly interact with the 3'UTRs of target mRNAs, thereby inhibiting mRNA translation or degrading mRNA (42). EcircRNAs that are primarily located in the cytoplasm can modulate the expression of their parental genes $(43,44)$, sponge miRNAs by acting as competitive endogenous RNAs (ceRNAs) $(25,31,43-46)$, attach miRNAs $(47,48)$, interact with or sponge RNA-binding proteins (RBPs) $(25,31,49-52)$, encode proteins $(25,31,43,53-55)$ and modulate protein translation (56-58) (Fig. 1).

The crosstalk between circRNAs and miRNAs is complex. CircRNAs commonly inhibit miRNA expression through a 'sponging' mechanism (25,31,41-44). Additionally, circ-CSNK1G3 and ci-RS-7 positively regulate the levels of miR-181b/d and miR-7, respectively $(45,46)$. Conversely, miR-200b negatively modulates the expression and function of circRNA-000839 (59). Whether miRNA, in turn, can regulate circRNA expression remains unknown. Moreover, the possibility that circRNAs also interact with each other has yet to be demonstrated. Furthermore, cytoplasmic circRNAs also modulate parental gene expression. For example, F-box and WD repeat domain-containing 7 (FBXW7), a well-known tumor suppressor gene, encodes the FBXW7 protein and circ-FBXW7. Circ-FBXW7 regulates the mRNA and protein levels of FBXW7 to repress the expression of c-Myc in a miRNA-dependent manner (41). Another study suggests that circ-filamin-binding LIM protein 1 (FBLIM1) positively regulates the expression of the FBLIM1 gene by sponging miR-346 (42).

CircRNAs regulate the activity and function of proteins in several ways, including via sponging, as a protein scaffold, by encoding proteins and modulating protein translation (Fig. 1). Circ-forkhead box O3 (Foxo3) can bind to both P21 and cyclin-dependent kinase 2 (CDK2) to generate ternary complexes that enhance the inhibitory effect of P21 on CDK2 (47). Circ-Foxo3 also functions as a protein scaffold, stabilizing Foxo3 protein expression by interacting with mouse double minute 2 (Mdm2) and P53 and inhibiting Mdm2-induced Foxo3 ubiquitination (50). Additionally, circ-Foxo3 inhibits the nuclear translocation of transcription factors, including HIF-1 $\alpha$, thereby inhibiting their 


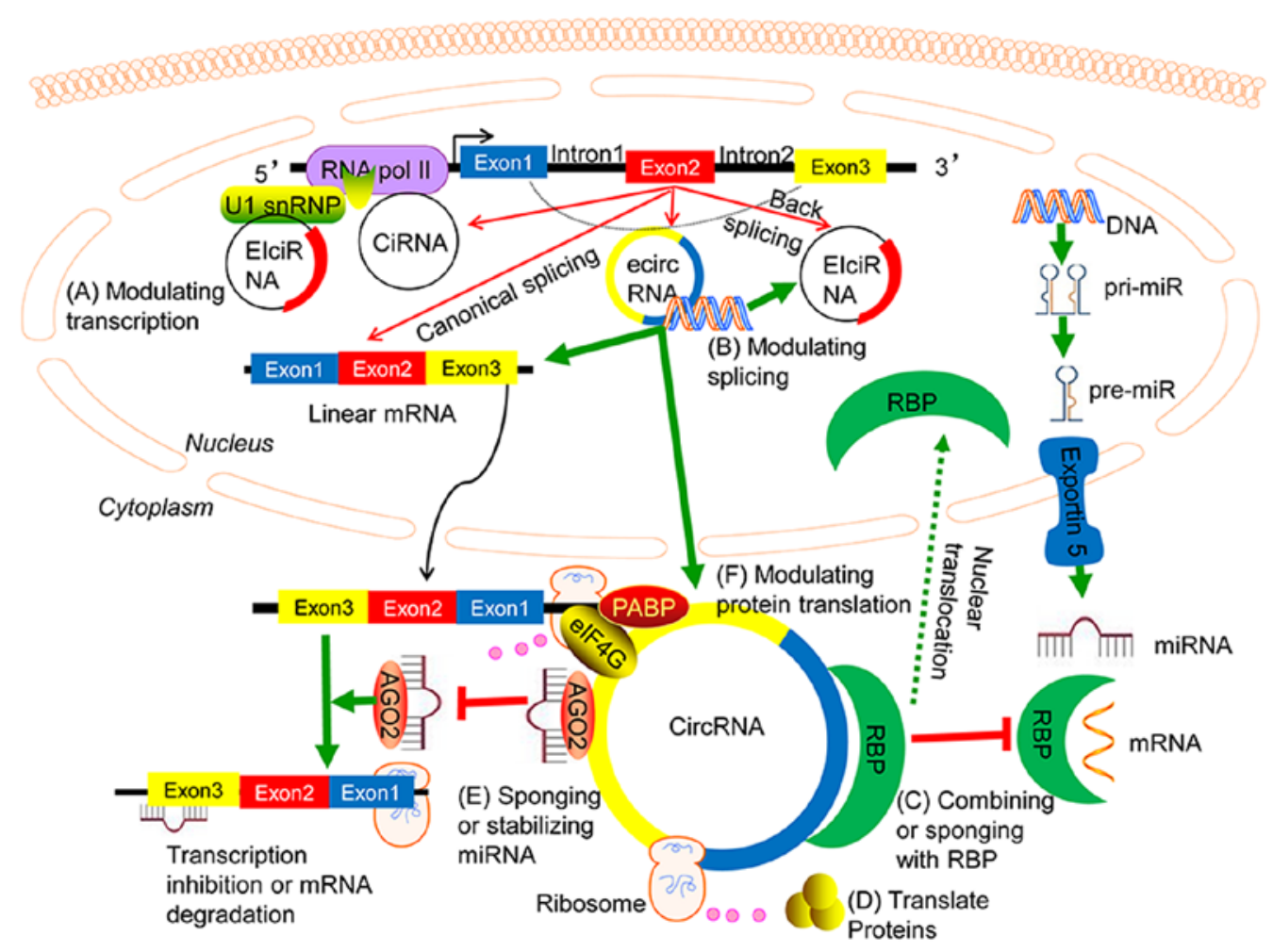

Figure 1. Biogenesis and function of circRNAs. In the nucleus, the circularization of at least one intron or one exon from a single gene gives rise to ciRNAs and ecircRNAs, respectively, whereas the formation of eiciRNA is based on the cyclization of at least one exon and one intron. CirRNAs or eiciRNA, mostly enriched in the nucleus, can modulate gene transcription and alternative splicing. EcircRNAs, which are primarily located in the cytoplasm, can modulate the expression of their parental genes, sponge miRNAs, interact with or sponge RBP, translate proteins and modulate protein translation. EcircRNAs positively regulate the expression of their target genes via sponging miRNAs to relieve the inhibitory effect of miRNAs on target genes. CircRNA, circular RNA; RBP, RNA-binding protein; ciRNA, intronic circRNA; ecircRNA, exonic circRNA; eiciRNA, exon-intron circRNA; miRNA, microRNA.

functions (49). Circ-ZKSCAN1 sponges fragile X mental retardation protein, preventing it from binding to its downstream target mRNA, cell cycle and apoptosis regulator 1, thereby attenuating the malignant biological behavior of hepatocellular carcinoma (HCC) through the Wnt signaling pathway (48). RBPs also regulate the formation of circRNAs. For example, RNA-binding motif protein 3 (RBM3) increases stearoyl-CoA desaturase-circ-RNA 2 synthesis to promote HCC cell proliferation (60), and Quaking 5 may promote the synthesis of circ-ZKSCAN1 (48). In addition, some circRNAs function through the proteins they encode. Circ- $\beta$-catenin encodes a 370 -amino-acid $\beta$-catenin, which inhibits glycogen synthase kinase $3 \beta$ (GSK $3 \beta)$-mediated $\beta$-catenin degradation and activates the Wnt/ $\beta$-catenin pathway in HCC (51). Circ-protein phosphatase 1 regulatory subunit 12A (PPP1R12A)-73aa (a protein encoded by circ-PPP1R12A), but not circPPP1R12A itself, accelerates colon cancer growth and metastasis (52). Moreover, circ-FBXW7 encodes the FBXW7 185-amino-acid protein (FBXW7-185aa), and the synergistic action of FBXW7 and FBXW7-185aa stabilizes c-Myc and promotes oncogenesis and tumor progression (42). Lastly, partial circRNAs modulate protein translation in a protein-dependent manner. CircRHOT1 regulates the translation of nuclear receptor subfamily 2 group F member 6 (NR2F6) by activating Tat-interacting protein of $60 \mathrm{kDa}$ to the Nr2f6 gene promoter (54). Circ-YY1-associated protein 1 (YAP) regulates the initiation efficiency of YAP protein translation by interacting with Yap mRNA and the proteins eIF4G and PABP (55). More recently, Sun et al (58) reported that circMYBL2 could facilitate fms-related receptor tyrosine kinase 3 (FLT3) protein translation efficiency by recruiting polypyrimidine tract-binding protein 1 to bind to Flt3 mRNA.

Recently, Chen et al (47) suggested that $~ 90 \%$ of circRNAs have an independent regulatory role in cell proliferation, compared with their linear counterparts. Nevertheless, certain circRNAs have functions that are similar to linear RNAs $(61,62)$. Peroxisome proliferator-activated receptors and their associated circRNA, circ-5379-6, both suppress tumor progression (61). Yao et al (62) also reported that circ-ZKSCAN1 and its parental gene both inhibited cell growth through distinct signaling pathways.

\section{Specificroles of circRNA in IDD}

Biofunctions of circRNA in IDD. A growing body of evidence suggests that circRNAs are extensively involved in a multitude of chronic diseases, including osteoarthritis (63) and cancer (64), as well as cardiovascular (65), neurodegenerative $(66,67)$ and immunological $(68,69)$ diseases. However, the role of circRNA in IDD remains unclear. To the best of our knowledge, only nine upregulated and nine downregulated circRNAs have been identified in degenerative NP samples compared with normal NP samples, and the functions of 12 of these dysregulated circRNAs remain fairly poorly understood $(70,71)$. Using microarray data from the Gene Expression Omnibus (GEO) database, Zhu et al (72) 
predicted three circRNA-mediated regulatory pathways in IDD, namely, circRNA-102348/miR-185-5p/TGFB1/ FOS, circRNA-102399/miR-302a-3p/HIF1A and circRNA-100086/miR-509-3p/MAPK1. However, further investigation is needed to elucidate their potential role in IDD (72). Moreover, the detailed biofunctions of circRNAs that have been identified are listed in Table I and Fig. 2. The upregulated circRNAs except circ-0004099 contribute to the occurrence and progression of IDD, whereas circ-0004099 and all downregulated circRNAs function as IDD repressors. CircRNAs act as IDD repressors or enhancers through the regulation of several pathological processes, including NPC apoptosis, proliferation, mitophagy and senescence, as well as the dysregulation of MMP, ADAMTS, inflammatory cytokines and ECM expression.

The five described IDD repressors have diverse functions: i) Circ-semaphorin 4B (SEMA4B) enhances NPC proliferation; ii) circ-SEMA4B and circ-excision repair cross-complementation group 2 (ERCC2) suppress NPC senescence; iii) circ-growth factor receptor bound protein 10 (GRB10), circ-vacuolar ATPase assembly factor VMA21 (VMA21) and circ-ERCC2 suppress NPC apoptosis; iv) circ-SEMA4B, circ-0004099, circ-VMA21 and circ-ERCC2 promote ECM synthesis; v) circ-VMA21 and circ-ERCC2 suppress MMP or ADAMTS expression; vi) circ-ERCC2 facilitates mitophagy; and vii) circ-0004099 inhibits IC secretion (12-17). The only known IDD enhancer, circRNA-104670, not only represses NPC proliferation and the synthesis of ECM components, but also promotes NPC apoptosis and MMP2 expression (15). These abnormally expressed circRNAs mediate pathological processes through several signaling pathways, including apoptosis-related pathways and ECM-related pathways.

Circ-VMA21-mediated IDD repression. Circ-VMA21 was the first identified IDD-related circRNA (12), providing invaluable insight into the modulation of IDD pathogenesis by circRNAs. Circ-VMA21 is downregulated in degenerative NP samples from patients with IDD compared with NP samples from controls (12). Similarly, the expression of circ-VMA21 is reduced in NPCs treated with both TNF- $\alpha$ and IL-1 $\beta$ (12). The progression of IDD is associated with the aberrant expression of circ-VMA21 in degenerative and normal NP samples. More specifically, circ-VMA21 blocked the progression of IDD, and its downregulation limited its protective effect. Circ-VMA21 serves a protective role in human NPCs and rat NP tissues predominantly through apoptosis-related pathways and the ECM components metabolism-related pathways.

Circ-VMA21 positively modulates $X$ linked inhibitor-of-apoptosis protein (XIAP) and represses the expression of caspase (CASP) family members (CASP-3, CASP-7 and CASP-9), as well as a number of degrading metabolic enzymes (MMP-3, MMP-13, ADAMTS-4 and ADAMTS-5). Circ-VMA21 also promotes the expression of ECM components, including COL2 and ACAN, by sponging miR-200c (12,73). Luciferase reporter and RNA pull-down assays confirmed that circ-VMA21 has five effective binding sites in miR-200c (12,73).

Pfirrmann classification is the most common method used for the evaluation of IDD severity, according to magnetic resonance imaging grade (74). Circ-VMA21 markedly decreased the Pfirrmann grade of IDD following injection into rat IDs. Altogether, these studies suggested that the circ-VMA21/miR-200c/XIAP axis may be involved in the regulation of IDD pathogenesis, providing novel therapeutic targets for IDD.

$I D D$ repressor circ-GRB10. Lan et al (75) analyzed the microarray data of human lumbar IDD and uploaded it into the GEO database. Our previous study identified three abnormally expressed circRNAs by analyzing circRNA microarray data from the GEO database, of which two were upregulated (circ-FAM169A and circ-SETD2) and one was downregulated (circ-GRB10) (13). The expression of circ-GRB10 was downregulated in 20 degenerative NP samples from patients with IDD undergoing discectomy compared with 20 nondegenerative NP samples from patients with fresh traumatic lumbar fracture (13). Mechanistically, the circ-GRB10-mediated pathological process of IDD is miR-328-5p-dependent. Functionally, circ-GRB10 acts as an IDD repressor of NPC apoptosis under nutrient deprivation conditions by sequestering miR-328-5p and promoting Erb-B2 receptor tyrosine kinase 2 (ERBB2) expression in NPCs. Thus, circ-GRB10 downregulation could decrease NPC survival, leading to IDD onset and progression.

IDD repressor circ-0004099. Wang et al (14) categorized patients with IDD according to the Pfirrmann classification criteria (74). Patients with Pfirrmann grade I/II were assigned to a nondegenerative group, whereas those with Pfirrmann grade IV/V constituted the degeneration group. The authors collected six degenerative NP samples from the patients of the degenerative group who were undergoing spinal surgery and six nondegenerative NP samples from the patients of the nondegenerative group with vertebral fracture or scoliosis $(13,14)$. Using circRNA microarray, circRNA expression profiles in the twelve samples were examined (14). Circ-0004099 was the most frequently upregulated circRNA in the degenerative samples (14). Moreover, circ-0004099 expression was increased in NPCs in a dose and time-dependent manner following treatment with TNF- $\alpha$. It was also demonstrated that this effect was mediated by MAPK and the NF- $\kappa \mathrm{B}$ signaling pathways (14). Notably, circ-0004099 overexpression enhanced (rather than repressed) the expression of Sox9 and ECM proteins, such as ACAN and COL2. Circ-004099 also repressed (rather than enhanced) proinflammatory cytokine secretion (TNF- $\alpha$, IL-1 $\beta$ and prostaglandin E2), and these changes were reversed by miR-616-5p mimic 14). Sox9 is a chondrocyte-specific transcription factor that promotes COL2 and ACAN synthesis $(76,77)$. Wang et al (14) also confirmed that Sox9 was the direct target of miR-616-5p. In addition, luciferase reporter, RNA immunoprecipitation and RNA pull-down assays indicated that circ-0004099 could bind to miR-616-5p (14). Collectively, these research results reveal that the circ-0004099/miR-616-5p/Sox 9 axis might play a protective role in IDD.

$I D D$ repressor circ-SEMA4B. Consistent with Guo's method (13), Wang et al (16) also analyzed the same circRNA microarray data that was downloaded from the GEO database. Circ-SEMA4B expression was the most significantly downregulated circRNA in 45 IDD specimens and had 


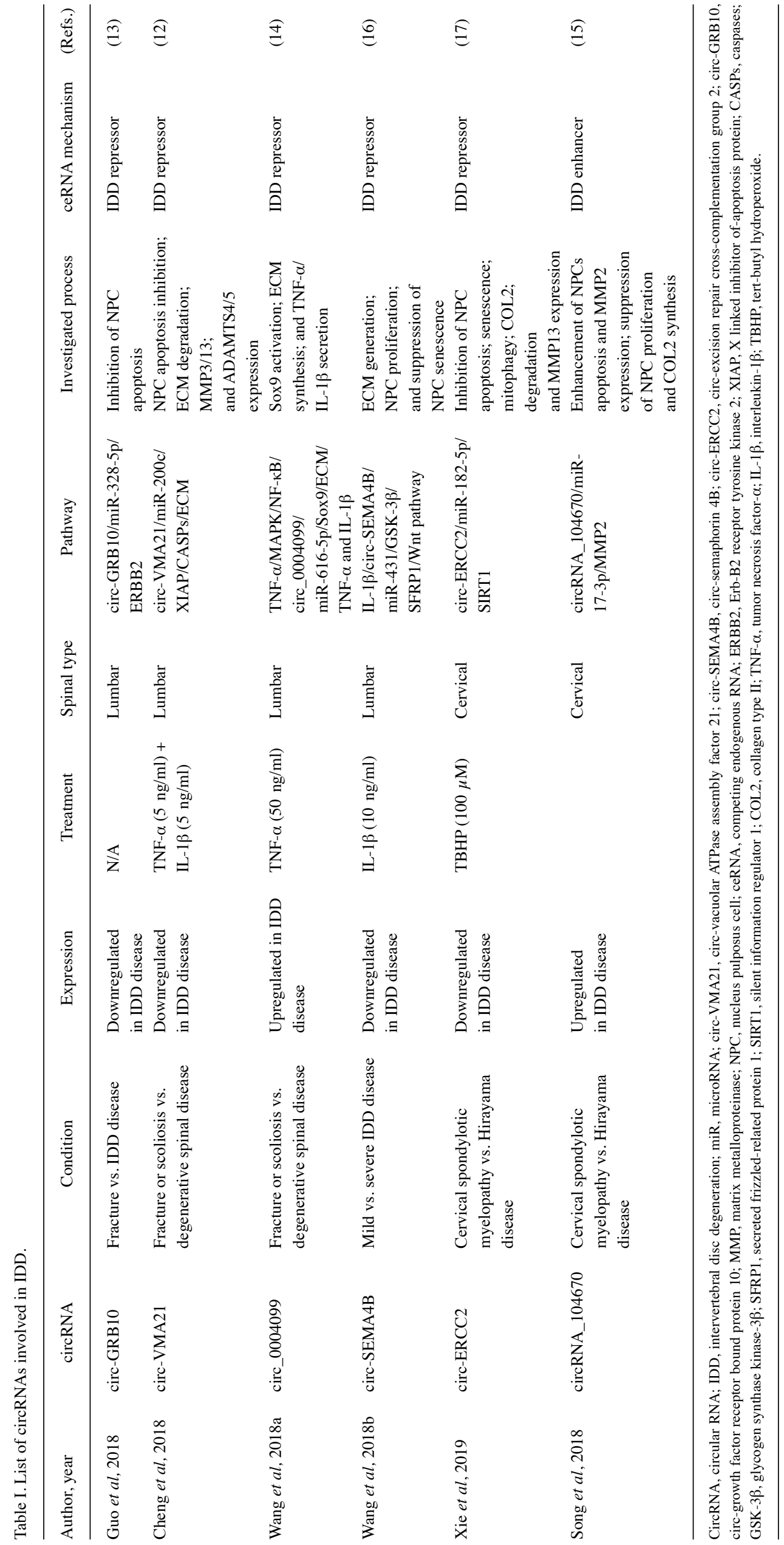




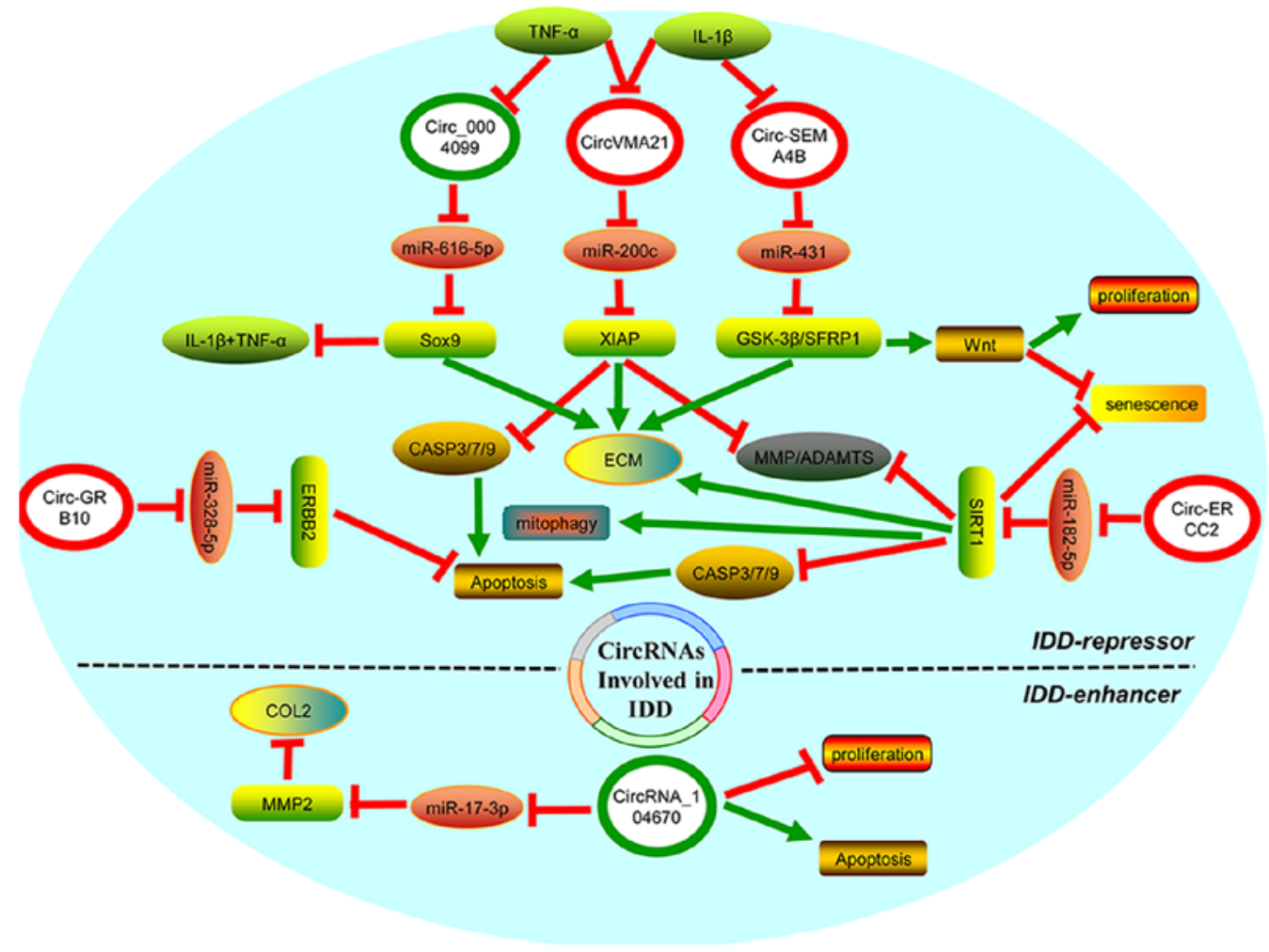

Figure 2. CircRNAs are involved in the regulation of IDD. In the upper half of the ellipse, circ-SEMA4B, circ-GRB10, circ-VMA21 and circ-ERCC2 were downregulated, whereas circ-0004099 was upregulated in IDD (green circle). All of them function as an IDD-repressor by regulating different miRNA-mRNA pathways, of which circ-SEMA4B, circ-0004099, circ-VMA21 and circ-ERCC2 promote the synthesis of key components of the ECM; circ-GRB10, circ-VMA21 and circ-ERCC2 suppress NPC apoptosis; circ-VMA21 and circ-ERCC2 suppress NPC senescence; circ-SEMA4B enhances NPC proliferation; circ-VMA21 and circ-ERCC2 suppress MMP or ADAMTS expression; circ-ERCC2 facilitates mitophagy; and circ-0004099 inhibits TNF- $\alpha$ and IL-1 $\beta$ secretion. Additionally, TNF- $\alpha$ can inhibit circ-0004099 expression, IL-1 $\beta$ can inhibit circ-SEMA4B expression, and both of them elevate the expression of circ-VMA21. In the lower half of the ellipse, circRNA-104670 was upregulated in IDD (green circle), which not only represses NPC proliferation and synthesis of ECM components, but also promotes NPC apoptosis and MMP2 expression via sponging miR-17-3p, thereby its acts as an IDD enhancer. CircRNA, circular RNA; IDD, intervertebral disc degeneration; circ-VMA21, circ-vacuolar ATPase assembly factor 21; circ-SEMA4B, circ-semaphorin 4B; circ-ERCC2, circ-excision repair cross-complementation group 2; circ-GRB10, circ-growth factor receptor bound protein 10; miR, microRNA; ECM, extracellular matrix; MMP, matrix metalloproteinase; NPC, nucleus pulposus cell; ADAMTS, ADAM metallopeptidases with thrombospondin type 1 motifs; TNF- $\alpha$, tumor necrosis factor- $\alpha$; IL-1 $\beta$, interleukin $1 \beta$.

a negative association with IDD severity, as bard on the Pfirrmann grade (16). Notably, circ-SEMA4B promoted the synthesis of ECM components and NPC proliferation, while inhibiting NPC senescence under IL-1 $\beta$ stimulation. It was also demonstrated that IL- $1 \beta$ exerted these effects by inhibiting circ-SEMA4B expression (16).

Previous studies have demonstrated that the Wnt signaling pathway serves an important role in the regulation of NPC proliferation and senescence $(78,79)$. For instance, circ-SEMA4B regulates the activation of the Wnt signaling pathway by sponging miR-431, the upstream regulator of two well-known Wnt signaling pathway inhibitors, GSK-3 $\beta$ and secreted frizzled-related protein 1 (SFRP1) $(16,80,81)$. Collectively, results from these studies suggest that circ-SEMA4B may be associated with the prognosis of patients with IDD and inhibits IDD development by regulating the miR-431/GSK-3 $\beta /$ SFRP1 axis.

IDD repressor circ-ERCC2. Xie et al (17) analyzed Song's (15) microarray analysis of circRNAs and Lan's (75) microarray dataset (GSE67566) and demonstrated that circ-ERCC2 was the most frequently downregulated circRNA in degenerative NP tissue $(15,74)$. Functional analyses also suggested that circ-ERCC2 modulated tert-butyl hydroperoxide-induced
NPC apoptosis (through CASP-3, CASP-7 and CASP-9), mitophagy (through PTEN-induced kinase 1, parkin, p62, and LC3II/I) and ECM structure (MMP13 and COL2) in vitro and in vivo (17). Fluorescence in situ hybridization and dual-luciferase assays demonstrated that circ-ERCC2 could bind to miR-182-5p (17).

Previous studies have indicated that silent mating type information regulator 2 homolog 1 (SIRT1) plays a significant role in mitophagy and apoptosis (82-84). SIRT1-small interfering (si)RNA inhibits NPC apoptosis and senescence. Moreover, this effect is suppressed by circ-ERCC2 and miR-182-5p inhibitor, suggesting that circERCC2 exerts a protective effect on NPCs that is dependent on miR-182-5p (17).

IDD enhancer circRNA_104670. CircRNA-104670 is related to cervical IDD and is upregulated $\sim 4.5$-fold in degenerative tissues from patients with cervical spondylotic myelopathy compared with normal tissues from patients with Hirayama disease (15). Furthermore, circRNA-104670 may represent a diagnostic marker for IDD. In a previous study, receiver operating characteristic curve analysis indicated that the area under the curve value of circRNA_104670 was 0.96 and the expression of circRNA_104670 was positively associated with 
Pfirrmann grade (15). Functionally, circRNA-104670 increased NPC apoptosis and suppressed NPC proliferation. Moreover, this circRNA also promoted MMP2 and repressed COL2 expression by sequestering miR-17-3p (15). Mice injected with circRNA_104670 siRNA presented lower Pfirrmann grades (15). Thus, circRNA-104670 may act as an IDD enhancer that regulates miR-17-3p and MMP2, leading to IDD progression.

\section{Current limitations and future directions}

All of the research on IDD-related circRNAs has shortcomings. Although a universal approach to NPC culture with inflammatory cytokine treatment and ID microenvironment stimulation has been developed, it does not account for the fact that different circRNAs have diverse affinities for various inflammatory cytokines. However, as highlighted in Table I, none of the investigations explain why they used IL-1 $\beta$ or TNF- $\alpha$ or both. Recently, Shen et al (63) approached this problem by detecting the expression of circRNAs in the cells stimulated with IL-1 $\beta$, TNF- $\alpha$ or both.

As the NP is a hypoxic environment, the potential role of HIF- $1 \alpha$ in IDD has been reviewed previously. In the development of IDD, HIF-1 $\alpha$ activation is involved in the regulation of IDD-related gene or protein expression (9). Thus, HIF-1 $\alpha$ is a crucial transcriptional regulator of IDD. Increasing evidence suggests that HIF-1 $\alpha$ is a target of ncRNAs in several diseases $(26,85,86)$. Nevertheless, hypoxia-related circRNA pathways in IDD are still poorly characterized.

Exosomes serve an important role in numerous physiological and pathological processes in various diseases $(33,87,88)$. Various factors, including HIF- $1 \alpha$, ncRNAs and proteins, among others, are present in exosomes (89). Thus, circRNAs contained within exosomes could serve as markers for diseases $(33,87,88)$. Importantly, whether HIF-1 $\alpha$ affects MMP and the ECM remains unclear (9). Nevertheless, we cannot rule out the possibility of the existence of the circRNA/HIF-1 $\alpha / \mathrm{MMP} / \mathrm{ECM}$ axis in the exosomes of IDD. Further research is needed to elucidate this.

The phosphoinositide 3-kinase (PI3K)/protein kinase B $(\mathrm{AKT}) / \mathrm{mechanistic}$ target of rapamycin (mTOR) axis regulates numerous biological events, including cell proliferation, apoptosis, metastasis and metabolism (90). Growing evidence also indicates that circRNA-mediated regulation of the $\mathrm{PI} 3 \mathrm{~K} / \mathrm{AKT} / \mathrm{mTOR}$ axis serves an essential role in the pathogenesis of several diseases, such as hepatocellular carcinoma and kidney cancer $(85,91,92)$. However, whether this holds true in IDD remains unclear. Recently, bioinformatics analysis predicted that circRNAs could regulate autophagy signaling pathways in IDD via sponging miRNAs (93). So, the role of circRNA-mediated autophagy in IDD cannot be ruled out and is a worthwhile research direction.

\section{Conclusions}

In conclusion, circRNAs function as ceRNAs to regulate the pathological process of IDD in a miRNA-dependent manner. However, circRNAs also have a number of other functions aside from their role as a ceRNA that have not been reported on in IDD. Whether circRNAs can sponge or interact with $\mathrm{RBP}$, encode proteins, modulate protein translation and gene expression in the context of IDD should also be addressed. Thus, understanding the biological role of circRNAs and their underlying molecular mechanism in the context of IDD would provide further insight into disease prevention strategies and contribute to the development of therapeutic targets for IDD.

\section{Acknowledgements}

Not applicable.

\section{Funding}

The present study was supported by grants from The National Natural Science Foundation of China (grant nos. 31670983 and 31900967) and The Natural Science Foundation of Tianjin City (grant no. 19JCQNJC09300).

\section{Availability of data and materials}

Not applicable.

\section{Authors' contributions}

YL, SZ and PP contributed to the concept and the design of the review. YL wrote the manuscript. XW helped draft the manuscript and drew the figures. LD provided significant suggestions for the study. ZH searched the literature and collated important reference information. BX critically reviewed the manuscript. All authors read and approved the final manuscript.

\section{Ethics approval and consent to participate}

Not applicable.

\section{Patient consent for publication}

Not applicable.

\section{Competing interests}

The authors declare that they have no competing interests.

\section{References}

1. Vos T, Abajobir AA, Abate KH, Abbafati C, Abbas KM, Abd-Allah F, Abdulkadre RS, Abdulle AM, Abebo TA, Abera SF, et al: Global, regional, and national incidence, prevalence, and years lived with disability for 328 diseases and injuries for 195 countries, 1990-2016: A systematic analysis for the global burden of disease study 2016. Lancet 390: 1211-1259, 2017.

2. Deyo RA and Tsui-Wu YJ: Descriptive epidemiology of low-back pain and its related medical care in the United States. Spine (Phila Pa 1976) 12: 264-268, 1987.

3. Risbud MV and Shapiro IM: Role of cytokines in intervertebral disc degeneration: Pain and disc content. Nat Rev Rheumatol 10: 44-56, 2014.

4. Battié MC, Videman T and Parent E: Lumbar disc degeneration: Epidemiology and genetic influences. Spine (Phila Pa 1976) 29: 2679-2690, 2004.

5. Humzah MD and Soames RW: Human intervertebral disc: Structure and function. Anat Rec 220: 337-356, 1988.

6. Sakai D and Andersson GB: Stem cell therapy for intervertebral disc regeneration: Obstacles and solutions. Nat Rev Rheumatol 11: 243-256, 2015. 
7. Jacobs WC, van der Gaag NA, Kruyt MC, Tuschel A, de Kleuver M, Peul WC, Verbout AJ and Oner FC: Total disc replacement for chronic discogenic low back pain: A cochrane review. Spine (Phila Pa 1976) 38: 24-36, 2013.

8. Skvortsova K, Iovino N and Bogdanović O: Functions and mechanisms of epigenetic inheritance in animals. Nat Rev Mol Cell Biol 19: 774-790, 2018.

9. Frapin L, Clouet J, Delplace V, Fusellier M, Guicheux J and Le Visage C: Lessons learned from intervertebral disc pathophysiology to guide rational design of sequential delivery systems for therapeutic biological factors. Advanced Drug Delivery Reviews 149-150: 49-71, 2019.

10. Fontana G, See E and Pandit A: Current trends in biologics delivery to restore intervertebral disc anabolism. Adv Drug Deliv Rev 84: 146-158, 2015.

11. Vergroesen PP, Kingma I, Emanuel KS, Hoogendoorn RJ, Welting TJ, van Royen BJ, van Dieën JH and Smit TH: Mechanics and biology in intervertebral disc degeneration: A vicious circle. Osteoarthritis Cartilage 23: 1057-1070, 2015

12. Cheng X, Zhang L, Zhang K, Zhang G, Hu Y, Sun X, Zhao C, $\mathrm{Li} \mathrm{H,} \mathrm{Li} \mathrm{YM} \mathrm{and} \mathrm{Zhao} \mathrm{J:} \mathrm{Circular} \mathrm{RNA} \mathrm{VMA21} \mathrm{protects} \mathrm{against}$ intervertebral disc degeneration through targeting miR-200c and X linked inhibitor-of-apoptosis protein. Ann Rheum Dis 77: 770-779, 2018

13. Guo W, Zhang B, Mu K, Feng SQ, Dong ZY, Ning GZ, Li HR, Liu S, Zhao L, Li Y, et al: Circular RNA GRB10 as a competitive endogenous RNA regulating nucleus pulposus cells death in degenerative intervertebral disk. Cell Death Dis 9: 319, 2018.

14. Wang H, He P, Pan H, Long J, Wang J, Li Z, Liu H, Jiang W and Zheng Z: Circular RNA circ-4099 is induced by TNF- $\alpha$ and regulates ECM synthesis by blocking miR-616-5p inhibition of Sox 9 in intervertebral disc degeneration. Exp Mol Med 50: 27, 2018.

15. Song J, Wang HL, Song KH, Ding ZW, Wang HL, Ma XS, Lu FZ, Xia XL, Wang YW, Fei-Zou and Jiang JY: CircularRNA_104670 plays a critical role in intervertebral disc degeneration by functioning as a ceRNA. Exp Mol Med 50: 94, 2018

16. Wang X, Wang B, Zou M, Li J, Lü G, Zhang Q, Liu F and Lu C: CircSEMA4B targets miR-431 modulating IL-1 $\beta$-induced degradative changes in nucleus pulposus cells in intervertebral disc degeneration via Wnt pathway. Biochim Biophys Acta Mol Basis Dis 1864: 3754-3768, 2018.

17. Xie L, Huang W, Fang Z, Ding F, Zou F, Ma X, Tao J, Guo J, Xia X, Wang H, et al: CircERCC2 ameliorated intervertebral disc degeneration by regulating mitophagy and apoptosis through miR-182-5p/SIRT1 axis. Cell Death Dis 10: 751, 2019.

18. Sanger HL, Klotz G, Riesner D, Gross HJ and Kleinschmidt AK Viroids are single-stranded covalently closed circular RNA molecules existing as highly base-paired rod-like structures. Proc Natl Acad Sci USA 73: 3852-3856, 1976.

19. Grabowski PJ, Zaug AJ and Cech TR: The intervening sequence of the ribosomal RNA precursor is converted to a circular RNA in isolated nuclei of Tetrahymena. Cell 23 467-476, 1981

20. Nigro JM, Cho KR, Fearon ER, Kern SE, Ruppert JM, Oliner JD, Kinzler KW and Vogelstein B: Scrambled exons. Cell 64: 607-613, 1991

21. Capel B, Swain A, Nicolis S, Hacker A, Walter M, Koopman P, Goodfellow P and Lovell-Badge R: Circular transcripts of the testis-determining gene Sry in adult mouse testis. Cell 73: 1019-1030, 1993.

22. Burd CE, Jeck WR, Liu Y, Sanoff HK, Wang Z and Sharpless NE: Expression of linear and novel circular forms of an INK4/ARF associated non-coding RNA correlates with atherosclerosis risk. PLoS Genet 6: e1001233, 2010.

23. Morris KV and Mattick JS: The rise of regulatory RNA. Nat Rev Genet 15: 423-437, 2014

24. Jeck WR and Sharpless NE: Detecting and characterizing circular RNAs. Nat Biotechnol 32: 453-461, 2014.

25. Li X, Yang L and Chen LL: The biogenesis, functions, and challenges of circular RNAs. Mol Cell 71: 428-442, 2018.

26. Boeckel JN, Jaé N, Heumüller AW, Chen W, Boon RA, Stellos K, Zeiher AM, John D, Uchida S and Dimmeler S: Identification and characterization of hypoxia-regulated endothelial circular RNA. Circ Res 117: 884-890, 2015.

27. Vicens Q and Westhof E: Biogenesis of circular RNAs. Cell 159: 13-14, 2014

28. Jeck WR, Sorrentino JA, Wang K, Slevin MK, Burd CE, Liu J, Marzluff WF and Sharpless NE: Circular RNAs are abundant, conserved, and associated with ALU repeats. RNA 19: 141-157, 2013.
29. Suzuki H,Zuo Y, Wang J, Zhang MQ, Malhotra A and Mayeda A Characterization of RNase R-digested cellular RNA source that consists of lariat and circular RNAs from pre-mRNA splicing. Nucleic Acids Res 34: e63, 2006

30. Vo JN, Cieslik M, Zhang Y, Shukla S, Xiao L, Zhang Y, Wu YM, Dhanasekaran SM, Engelke CG, Cao X, et al: The Landscape of circular RNA in cancer. Cell 176: 869-881.e13, 2019.

31. Patop IL, Wüst S and Kadener S: Past, present, and future of circRNAs. EMBO J 38: e100836, 2019.

32. Salzman J, Chen RE, Olsen MN, Wang PL and Brown PO: Cell-type specific features of circular RNA expression. PLoS Genet 9: e1003777, 2013.

33. Wang Y, Liu J, Ma J, Sun T, Zhou Q, Wang W, Wang G, Wu P, Wang $\mathrm{H}$, Jiang L, et al: Exosomal circRNAs: Biogenesis, effect and application in human diseases. Mol Cancer 18: 116, 2019.

34. Li Y, Zheng Q, Bao C, Li S, Guo W, Zhao J, Chen D, Gu J, He X and Huang S: Circular RNA is enriched and stable in exosomes: A promising biomarker for cancer diagnosis. Cell Res 25 981-984, 2015.

35. Schmidt CA, Giusto JD, Bao A, Hopper AK and Matera AG: Molecular determinants of metazoan tricRNA biogenesis. Nucleic Acids Res 47: 6452-6465, 2019.

36. Guarnerio J, Bezzi M, Jeong JC, Paffenholz SV, Berry K, Naldini MM, Lo-Coco F, Tay Y, Beck AH and Pandolfi PP Oncogenic role of fusion-circRNAs derived from cancer-associated chromosomal translocations. Cell 165: 289-302, 2016.

37. Liu X, Wang X, Li J, Hu S, Deng Y, Yin H, Bao X, Zhang QC, Wang G, Wang B, et al: Identification of mecciRNAs and their roles in mitochondrial entry of proteins. Sci China Life Sci: Jan 21, 2020 (Epub ahead of print).

38. Li Z, Huang C, Bao C, Chen L, Lin M, Wang X, Zhong G, $\mathrm{Yu} \mathrm{B}, \mathrm{Hu}$ W, Dai L, et al: Exon-intron circular RNAs regulate transcription in the nucleus. Nat Struct Mol Biol 22: 256-264, 2015.

39. Zhang Y, Zhang XO, Chen T, Xiang JF, Yin QF, Xing YH, Zhu S, Yang $L$ and Chen LL: Circular intronic long noncoding RNAs. Mol Cell 51: 792-806, 2013

40. Ashwal-Fluss R, Meyer M, Pamudurti NR, Ivanov A, Bartok O, Hanan M, Evantal N, Memczak S, Rajewsky N and Kadener S: circRNA biogenesis competes with pre-mRNA splicing. Mol Cell 56: 55-66, 2014.

41. Graves P and Zeng Y: Biogenesis of mammalian MicroRNAs: A global view. Genomics Proteomics Bioinformatics 10: 239-245, 2012.

42. Bartel DP: MiRNAs: Target recognition and regulatory functions. Cell 136: 215-233, 2009.

43. Ye F, Gao G, Zou Y, Zheng S, Zhang L, Ou X, Xie X and Tang H: circFBXW7 inhibits malignant progression by sponging miR-197-3p and encoding a 185-aa protein in triple-negative breast cancer. Mol Ther Nucleic Acids 18: 88-98, 2019.

44. Bai N, Peng E, Qiu X, Lyu N, Zhang Z, Tao Y, Li X and Wang Z: circFBLIM1 act as a ceRNA to promote hepatocellular cancer progression by sponging miR-346. J Exp Clin Cancer Res 37: 172,2018

45. Zhang PF, Wei CY, Huang XY, Peng R, Yang X, Lu JC, Zhang C, Gao C, Cai JB, Gao PT, et al: Circular RNA circTRIM33-12 acts as the sponge of MicroRNA-191 to suppress hepatocellular carcinoma progression. Mol Cancer 18: 105, 2019.

46. Hansen TB, Jensen TI, Clausen BH, Bramsen JB, Finsen B, Damgaard CK and Kjems J: Natural RNA circles function as efficient microRNA sponges. Nature 495: 384-388, 2013.

47. Chen S, Huang V, Xu X, Livingstone J, Soares F, Jeon J, Zeng Y, Hua JT, Petricca J, Guo H, et al: Widespread and functional RNA circularization in localized prostate cancer. Cell 176: 831-843. e22, 2019.

48. Piwecka M, Glazar P, Hernandez-Miranda LR, Memczak S, Wolf SA, Rybak-Wolf A, Filipchyk A, Klironomos F, Cerda Jara CA, Fenske P, et al: Loss of a mammalian circular RNA locus causes miRNA deregulation and affects brain function. Science 357: eaam8526, 2017.

49. Du WW, Yang W, Liu E, Yang Z, Dhaliwal P and Yang BB: Foxo3 circular RNA retards cell cycle progression via forming ternary complexes with p21 and CDK2. Nucleic Acids Res 44: 2846-2858, 2016

50. Zhu YJ, Zheng B, Luo GJ, Ma XK, Lu XY, Lin XM, Yang S, Zhao Q, Wu T, Li ZX, et al: Circular RNAs negatively regulate cancer stem cells by physically binding FMRP against CCAR1 complex in hepatocellular carcinoma. Theranostics 9: 3526-3540, 2019. 
51. Du WW, Yang W, Chen Y, Wu ZK, Foster FS, Yang Z, Li X and Yang BB: Foxo3 circular RNA promotes cardiac senescence by modulating multiple factors associated with stress and senescence responses. Eur Heart J 38: 1402-1412, 2017.

52. Du WW, Fang L, Yang W, Wu N, Awan FM, Yang Z and Yang BB: Induction of tumor apoptosis through a circular RNA enhancing Foxo3 activity. Cell Death Differ 24: 357-370, 2017.

53. Liang WC, Wong CW, Liang PP, Shi M, Cao Y, Rao ST, Tsui SK, Waye MM, Zhang Q, Fu WM and Zhang JF: Translation of the circular RNA circ $\beta$-catenin promotes liver cancer cell growth through activation of the Wnt pathway. Genome Biol 20: 84, 2019.

54. Zheng X, Chen L, Zhou Y, Wang Q, Zheng Z, Xu B, Wu C, Zhou $\mathrm{Q}, \mathrm{Hu} \mathrm{W}, \mathrm{Wu} \mathrm{C}$ and Jiang J: A novel protein encoded by a circular RNA circPPP1R12A promotes tumor pathogenesis and metastasis of colon cancer via Hippo-YAP signaling. Mol Cancer 18: 47, 2019

55. Yang Y, Gao X, Zhang M, Yan S, Sun C, Xiao F, Huang N, Yang X, Zhao K, Zhou H, et al: Novel role of FBXW7 circular RNA in repressing glioma tumorigenesis. J Natl Cancer Inst 110: 304-315, 2018

56. Wang L, Long H, Zheng Q, Bo X, Xiao X and Li B: Circular RNA circRHOT1 promotes hepatocellular carcinoma progression by initiation of NR2F6 expression. Mol Cancer 18: 119, 2019.

57. Wu N, Yuan Z, Du KY, Fang L, Lyu J, Zhang C, He A, Eshaghi E, Zeng K, Ma J, et al: Translation of yes-associated protein (YAP) was antagonized by its circular RNA via suppressing the assembly of the translation initiation machinery. Cell Death Differ 26: 2758-2773, 2019.

58. Sun YM, Wang WT, Zeng ZC, Chen TQ, Han C, Pan Q, Huang W, Fang K, Sun LY, Zhou YF, et al: CircMYBL2, a circRNA from, MYBL2 regulates FLT3 translation by recruiting PTBP1 to promote FLP3-ITD AML progression. Blood 134: 1533-1546, 2019.

59. Wang BG, Li JS, Liu YF and Xu Q: MicroRNA-200b suppresses the invasion and migration of hepatocellular carcinoma by downregulating RhoA and circRNA_000839. Tumour Biol 39: $1010428317719577,2017$.

60. Dong W, Dai ZH, Liu FC, Guo XG, Ge CM, Ding J, Liu H and Yang F: The RNA-binding protein RBM3 promotes cell proliferation in hepatocellular carcinoma by regulating circular RNA SCD-circRNA 2 production. EBioMedicine 45: 155-167, 2019.

61. Zhang N, Li G, Li X, Xu L and Chen M: Circ5379-6, a circular form of tumor suppressor PPAR $\alpha$, participates in the inhibition of hepatocellular carcinoma tumorigenesis and metastasis. Am J Transl Res 10: 3493-3503, 2018.

62. Yao Z, Luo J, Hu K, Lin J, Huang H, Wang Q, Zhang P, Xiong Z, He C, Huang Z, et al: ZKSCAN1 gene and its related circular RNA (circZKSCAN1) both inhibit hepatocellular carcinoma cell growth, migration, and invasion but through different signaling pathways. Mol Oncol 11: 422-437, 2017.

63. Shen S, Wu Y, Chen J, Xie Z, Huang K, Wang G, Yang Y, Ni W, Chen Z, Shi P, et al: CircSERPINE2 protects against osteoarthritis by targeting miR-1271 and ETS-related gene. Ann Rheum Dis 78: 826-836, 2019.

64. Shang Q, Yang Z, Jia R and Ge S: The novel roles of circRNAs in human cancer. Mol Cancer 18: 6, 2019.

65. Aufiero S, Reckman YJ, Pinto YM and Creemers EE: Circular RNAs open a new chapter in cardiovascular biology. Nat Rev Cardiol 16: 503-514, 2019.

66. Akhter R: Circular RNA and Alzheimer's disease. Adv Exp Med Biol 1087: 239-243, 2018.

67. Floris G, Zhang L, Follesa P and Sun T: Regulatory role of circular RNAs and neurological disorders. Mol Neurobiol 54: 5156-5165, 2017.

68. Chen X, Yang T, Wang W, Xi W, Zhang T, Li Q, Yang A and Wang T: Circular RNAs in immune responses and immune diseases. Theranostics 9: 588-607, 2019.

69. Yang L, Fu J and Zhou Y: Circular RNAs and their emerging roles in immune regulation. Front Immunol 9: 2977, 2018.

70. Zou F, Ding Z, Jiang J, Lu F, Xia X and Ma X: Confirmation and preliminary analysis of circRNAs potentially involved in human intervertebral disc degeneration. Mol Med Rep 16: 9173-9180, 2017.

71. Wang S, Sun J, Yang H, Zou W, Zheng B, Chen Y, Guo Y and Shi J: Profiling and bioinformatics analysis of differentially expressed circular RNAs in human intervertebral disc degeneration. Acta Biochim Biophys Sin (Shanghai) 51: 571-579, 2019.

72. Zhu J, Zhang $X$, Gao W, Hu H, Wang $X$ and Hao D IncRNA/circRNA-miRNA-mRNA ceRNA network in lumbar intervertebral disc degeneration. Mol Med Rep 20: 3160-3174, 2019.

73. Collison J: Degenerative disc disease: Circular RNA reduces cell death in IVD disease. Nat Rev Rheumatol 14: 123, 2018.
74. Pfirrmann CW, Metzdorf A, Zanetti M, Hodler J and Boos N: Magnetic resonance classification of lumbar intervertebral disc degeneration. Spine (Phila. PA 1976) 26: 1873-1878, 2001.

75. Lan PH, Liu ZH, Pei YJ, Wu ZG, Yu Y, Yang YF, Liu X, Che L, Ma CJ, Xie YK, et al: Landscape of RNAs in human lumbar disc degeneration. Oncotarget 7: 63166-63176, 2016.

76. Sekiya I, Tsuji K, Koopman P, Watanabe H, Yamada Y, Shinomiya K, Nifuji A and Noda M: SOX9 enhances aggrecan gene promoter/enhancer activity and is up-regulated by retinoic acid in a cartilage-derived cell line, TC6. J Biol Chem 275: 10738-10744, 2000.

77. Lefebvre V, Huang W, Harley VR, Goodfellow PN and de Crombrugghe B: SOX9 is a potent activator of the chondrocyte-specific enhancer of the pro alpha1(II) collagen gene. Mol Cell Biol 17: 2336-2346, 1997.

78. Hiyama A, Sakai D, Risbud MV, Tanaka M, Arai F, Abe K and Mochida J: Enhancement of intervertebral disc cell senescence by $\mathrm{WNT} / \beta$-catenin signaling induced matrix metalloproteinase expression. Arthritis Rheum 62: 3036-3047, 2010.

79. Hiyama A, Sakai D, Tanaka M, Arai F, Nakajima D, Abe K and Mochida J: The relationship between the Wnt/ $/$-catenin and TGF- $\beta$ /BMP signals in the intervertebral disc cell. J Cell Physiol 226: 1139-1148, 2011.

80. Guezguez B, Almakadi M, Benoit YD, Shapovalova Z, Rahmig S, Fiebig-Comyn A, Casado FL, Tanasijevic B, Bresolin S, Masetti R, et al: GSK3 deficiencies in hematopoietic stem cells initiate pre-neoplastic state that is predictive of clinical outcomes of human acute leukemia. Cancer Cell 29: 61-74, 2016.

81. Tao J, Abudoukelimu M, Ma YT, Yang YN, Li XM, Chen BD, Liu F, He CH and Li HY: Secreted frizzled related protein 1 protects H9C2 cells from hypoxia/re-oxygenation injury by blocking the Wnt signaling pathway. Lipids Health Dis 15: 72, 2016.

82. Guo J, Shao M, Lu F, Jiang J and Xia X: Role of Sirtl plays in nucleus pulposus cells and intervertebral disc degeneration. Spine (Phila Pa 1976) 42: E757-E766, 2017.

83. Wang D, Hu Z, Hao J, He B, Gan Q, Zhong X, Zhang X, Shen J, Fang J and Jiang W: SIRT1 inhibits apoptosis of degenerative human disc nucleus pulposus cells through activation of Akt pathway. Age (Dordr) 35: 1741-1753, 2013.

84. Yao ZQ, Zhang X, Zhen Y, He XY, Zhao S, Li XF, Yang B, Gao F, Guo FY, Fu L, et al: A novel small-molecule activator of Sirtuin-1 induces autophagic cell death/mitophagy as a potential therapeutic strategy in glioblastoma. Cell Death Dis 9: 767, 2018.

85. Wei Y, Chen X, Liang C, Ling Y, Yang X, Ye X, Zhang H, Yang P, Cui X, Ren Y, et al: A noncoding regulatory RNAs network driven by Circ-CDYL acts specifically in the early stages hepatocellular carcinoma. Hepatology 71: 130-147, 2020.

86. Ren S, Liu J, Feng Y, Li Z, He L, Li L, Cao X, Wang Z and Zhang Y: Knockdown of circDENND4C inhibits glycolysis, migration and invasion by up-regulating $\mathrm{miR}-200 \mathrm{~b} / \mathrm{c}$ in breast cancer under hypoxia. J Exp Clin Cancer Res 38: 388, 2019.

87. Ren GL, Zhu J, Li J and Meng XM: Noncoding RNAs in acute kidney injury. J Cell Physiol 234: 2266-2276, 2019.

88. Chaichian S, Shafabakhsh R, Mirhashemi SM, Moazzami B and Asemi Z: Circular RNAs: A novel biomarker for cervical cancer. J Cell Physiol 235: 718-724, 2020.

89. Braicu C, Tomuleasa C, Monroig P, Cucuianu A, Berindan-Neagoe I and Calin GA: Exosomes as divine messengers: Are they the hermes of modern molecular oncology? Cell Death Differ 22: 34-45, 2015.

90. Engelman JA, Luo J and Cantley LC: The evolution of phosphatidylinositol 3-kinases as regulators of growth and metabolism. Nat Rev Genet 7: 606-619, 2006.

91. Liu Y, Hou J, Zhang M, Seleh-Zo E, Wang J, Cao B and An X: circ-016910 sponges miR-574-5p to regulate cell physiology and milk synthesis via MAPK and PI3K/AKT-mTOR pathways in GMECs. J Cell Physiol 235: 4198-4216, 2020.

92. Chen T, Yu Q, Xin L and Guo L: Circular RNA circC3P1 restrains kidney cancer cell activity by regulating miR-21/PTEN axis and inactivating PI3K/AKT and NF-kB pathways. J Cell Physiol 235: 4001-4010, 2020 .

93. Xu G, Liu C, Jiang J, Liang T, Yu C, Qin Z, Zhang Z, Lu Z and Zhan X: A novel mechanism of intervertebral disc degeneration: Imbalance between autophagy and apoptosis. Epigenomics: Apr 14, 2020 (Epub ahead of print).

This work is licensed under a Creative Commons Attribution-NonCommercial-NoDerivatives 4.0 International (CC BY-NC-ND 4.0) License. 